\title{
The effect of moderate weight loss on echocardiographic parameters in obese female patients
}

\author{
Z. Sidó, ${ }^{1}$ P. Jákó, ${ }^{1}$ G. Pavlik ${ }^{2}$ \\ ${ }^{1}$ National Institute for Sports Medicine, Department of Conditioning and Internal Medicine, \\ Budapest, Hungary \\ ${ }^{2}$ Semmelweis University, Faculty of Physical Education and Sports Sciences, \\ Department of Health Sciences and Sports Medicine, Budapest, Hungary \\ Received: September 1, 2000 \\ Accepted: January 2, 2001 \\ Based on the data collected by KNOLL Hungary Ltd. in Hungary in 1999, 37\% of the \\ adult population is overweight while $23 \%$ is obese. Inappropriate diet containing excess calories \\ and physical inactivity are responsible for these statistical values. In their former studies, the \\ authors investigated the effects of different stages of obesity on the cardiovascular system, and \\ have verified that even moderate obesity elicits pathological geometric and functional changes in \\ the heart. \\ In the present study, effect of a half-year-long life-style modification program on the \\ morphologic and functional characteristics of the heart was investigated in twenty-one obese \\ women. Life-style modification contained a diet with reduced energy uptake (1000-1300 Cal/day) \\ and a regular physical training of minimum 3-4 hours weekly. By the end of the sixth month the \\ weight loss was $5.1 \mathrm{~kg}(5.9 \%)$ on an average. There was a marked reduction in cardiac \\ dimensions measured by echocardiography, with a very slight, non-significant decrease in left \\ ventricular internal diameter, and a marked, significant reduction in the left ventricular wall \\ thickness. Decrease of the left ventricular muscle mass exceeded the decrease of body weight. A \\ marked elevation was found in the E/A quotient that reflected a definite improvement in diastolic \\ function. \\ Results indicate that physical training programs have a favourable effect on the \\ echocardiographic parameters, therefore the process is reversible even without a pharmacological \\ intervention.
}

Correspondence should be addressed to

Gábor Pavlik

Semmelweis University

Faculty of Physical Education and Sports Sciences,

Department of Health Sciences and Sports Medicine

H-1123 Budapest, Alkotás u. 44, Hungary 
Keywords: obesity, physical exercise, echocardiography, physical inactivity, reduced energy uptake, Life-style modification, left ventricular internal diameter, left ventricular wall thickness

Obesity in adulthood has been confirmed to correlate closely with cardiovascular morbidity and mortality $(2,4,23)$. This point has special importance because $20 \%$ of the male population and nearly $30 \%$ of the female population are obese in the economically developed countries. Data collected in our country by KNOLL Hungary Ltd. in 1999 demonstrate that $37 \%$ of the adult population is overweight, and $23 \%$ is obese. The Honolulu Heart Program and The Framingham Heart Study have confirmed that obesity acts as an independent factor in coronary heart disease, in congestive heart failure, in stroke as well as in cardiovascular mortality $(1,24)$. It is also widely known that obesity is often accompanied by hypertension, reduced glucose-tolerance, abnormalities of the lipid metabolism, atherosclerosis and a special type of cardiomyopathy, and these symptoms are referred to as the common name of multimetabolic X-syndrome $(19,20,25)$.

We are acquainted with the fact that physical inactivity is a risk factor that may give rise to excess body weight and obesity $(5,11,37,47)$. Hungarian surveys have revealed that nearly $70 \%$ of the adult population lives a life that lacks physical activity (42). Inappropriate diet containing excess calories and physical inactivity lurk behind these statistical values of obesity in our country. Our study has also confirmed the general observation that the physical performance capacity of obese people is significantly lower than that of healthy individuals of normal body weight (47). At the same time it is also known that intense physical activity plays a favourable role both in the prevention and the management of obesity and cardiovascular diseases $(5,7)$.

The echocardiographic changes are well defined in several cardiovascular diseases (e.g. hypertension, cardiomyopathy, etc.) (3, 12, 17, 24, 45). However, only few authors have dealt with the echocardiographic examinations of obese patients, the data available are rather limited.

In our former studies we investigated the effects of different stages of obesity on the cardiovascular system, and we have verified that even obesity of an intermediate stage is able to elicit pathological geometrical and functional changes in the heart. We have observed that obesity does not only result in the increase of the size of the left ventricle and atrium, but it also affects the right side of the heart. Our observations revealed that marked accumulation of pericardial fat tissue was also quite common and it jeopardised the relaxation of the heart. We concluded that severe obesity that had been present for a longer period put a great load on the heart and induced eccentric left ventricular hypertrophy resulting eventually in diastolic dysfunction in spite of an intact systolic function. Systolic left ventricular function deteriorates only at a later stage of obesity (42-45). 
The management of obesity is a long-winded, difficult task for both patient and physician. In our days it is widely accepted that the cornerstones of management are controlled diet and increased physical activity and psychic guidance (7, 11, 16, 20, 21, $22,41,46,48)$.

The aim of the present work was to investigate the effects of a six-month life-style modification program on the body dimensions, cardiac measures and cardiac functions in obese women. Life-style modification consisted of regular physical training and of some dietary restrictions.

\section{Materials and Methods}

Twenty-one obese women participated in the study. Basic information on the participants can be seen in Table I. Diabetes and hypertension were not seen among the participants.

Patients participating in the program kept to a 1000-1300 Cal/day diet divided into 4-5 daily meals during the half-year study period. Physical exercise (walking, gymnastics, swimming, etc.) was usually performed at medium intensity (pulse rate during physical strain was in the range of 120-140/min) 3-4 times per week. Patients reported for body weight check-ups at the end of the first, third and sixth months.

Echocardiographic examinations using a Vingmed System V device with a 2.5 MHz transducer were performed on two occasions between July 1999 and May 2000. The two-dimensional echo-guided M-mode images were recorded in the parasternal short- and long-axis planes, and measurements were also performed on the two- and four-chamber apical images. The measured and calculated echocardiographic parameters were as follows. Aortic diameter (AOD), left atrial parasternal diameter (LAD1), interventricular septal diastolic thickness (IVSDT), left ventricular posterior wall diastolic thickness (LVPWDT), left ventricular internal diastolic diameter (LVIDD), right atrial longitudinal diameter (RAD), left atrial longitudinal diameter (LAD2), left ventricular longitudinal diastolic diameter (LVLDD) and left ventricular muscle mass (LVM). Left ventricular mass was calculated by the formula elaborated by Devereux and Reichek: LVM $=1.04 \times\left[(\text { LVIDD+IVSDT+PWDT })^{3}-(\text { LVIDD })^{3}-13.6\right]$ (9). The software in the echocardiograph calculated the ejection fraction (EF) of the left ventricle by Teichholz. 
Table I

Basic antropometric and personal data of participants (mean \pm S.D.)

\begin{tabular}{lr}
\hline & $\mathrm{n}=21$ \\
\hline Height $(\mathrm{cm})$ & $165.1 \pm 5.10$ \\
Age (year) & $44.0 \pm 8.00$ \\
Obesity (year) & $10.3 \pm 0.78$ \\
BSA $\left(\mathrm{m}^{2}\right)$ & $1.91 \pm 0.05$ \\
\hline
\end{tabular}

The transmitral Doppler flow-curve helps us in determining early diastolic peak filling velocity (E) reflecting the diastolic function, peak velocity reflecting the contraction of the left atrium (A), the ratio of these two velocities (E/A), and isovolumetric relaxation time (IVRT). Patient figures were obtained by taking the average of three measurements in all cases. The measurements and the calculations were performed according to the recommendations of the American Echocardiographic Association $(9,12,18,39)$. Data were processed on a computer; statistics were analysed by using the SPSS program, differences between pre- and post-training values were checked by $t$-tests for dependent samples.

\section{Results}

Body dimensions and indices are shown in Table II demonstrating that the women belonged to obesity group I by the basis of the international classification of BMI $(2,4)$. Patients displayed an average weight loss of $2.8 \mathrm{~kg}$ in the first month, and their body weight decreased by another $1.2 \mathrm{~kg}$ by the end of the third month, and the average $5.1 \mathrm{~kg}$ weight loss developed after six months. This weight loss was $5.9 \%$ of the initial body mass and was strongly significant. Decrease of BMI was quite obviously similar to the decrease in BM (5.5\%). This means that in spite of significant weight reduction, the previously obese patients still remained overweight. Their waist-to-hip ratio did not display any significant alteration.

Tables III and IV display the changes measured and calculated in the echocardiographic parameters. Left ventricular wall thickness, namely, IVSDT and LVPWDT, the longitudinal diameter of the left ventricle (LVLDD), the atrium-apex diameters (RAD and LAD2) and LVM decreased significantly, while there was no significant change in LVIDD, EF and in AOD. 


\section{Table II}

Changes of body dimensions and hemodynamic data (mean \pm S.D.)

\begin{tabular}{lcc}
\hline & \multicolumn{2}{c}{$(n=21)$} \\
\hline & Before & After \\
\hline Weight $(\mathrm{kg})^{* * *}$ & $86.6 \pm 12.4$ & $81.5 \pm 12.4$ \\
BMI $\left(\mathrm{kg} / \mathrm{m}^{2}\right)^{* * *}$ & $31.7 \pm 4.8$ & $30.0 \pm 4.6$ \\
Waist to hip ratio & $0.85 \pm 0.07$ & $0.83 \pm 0.07$ \\
Systolic pressure (mmHg) & $141 \pm 11$ & $132 \pm 9$ \\
Diastolic pressure $(\mathrm{mmHg})$ & $87 \pm 9$ & $79 \pm 6$ \\
Pulse rate (bpm) & $89 \pm 7$ & $76 \pm 6$ \\
\hline
\end{tabular}

$* * * \mathrm{p}<0.001$

From the left ventricular morphologic parameters, wall thickness showed a more marked decrease than LVIDD did; IVSDT and LVPWT were diminished by 8.4 and $8.2 \%$, respectively, while the decrease of LVIDD was only $3.5 \%$ and statistically not significant. Calculating from these two components, LVM displayed a very marked difference: a decrease of $18.8 \%$, the greatest change among the morphological parameters.

\section{Table III}

Left ventricular echocardiographic data before and after weight reduction (mean \pm S.D.)

\begin{tabular}{lrr}
\hline & $(n=21)$ \\
\hline & \multicolumn{3}{c}{ Before } & After \\
\hline IVSDT $(\mathrm{mm})^{* *}$ & $8.84 \pm 1.54$ & $8.10 \pm 1.07$ \\
LVPWDT (mm)*** & $9.7 \pm 1.2$ & $8.9 \pm 0.9$ \\
LVIDD $(\mathrm{mm})$ & $50.8 \pm 5.4$ & $49.1 \pm 5.0$ \\
LVLDD $(\mathrm{mm})^{*}$ & $77.6 \pm 7.5$ & $74.4 \pm 8.5$ \\
LVM $(\mathrm{g})^{* *}$ & $202.9 \pm 67.1$ & $164.8 \pm 41.5$ \\
EF $(\%)$ & $70.7 \pm 7.6$ & $73.4 \pm 10.4$ \\
\hline
\end{tabular}

IVSDT: interventricular septal diastolic thickness,

LVPWDT: left ventricular posterior wall diastolic thickness,

LVIDD: left ventricular internal diastolic diameter,

LVLDD: left ventricular longitudinal diastolic diameter,

LVM: left ventricular mass,

EF: ejection fraction,

$*: \mathrm{p}<0.05, * *: \mathrm{p}<0.01, * * *: \mathrm{p}<0.001$ 
Table IV

Aortic and atrial echocardiographic data before and after weight reduction (mean \pm S.D.)

\begin{tabular}{lcc}
\hline & \multicolumn{2}{c}{$(n=21)$} \\
\hline & Before & After \\
\hline AOD $(\mathrm{mm})$ & $27.27 \pm 2.56$ & $27.25 \pm 2.54$ \\
LAD1 (mm)** & $37.79 \pm 6.17$ & $36.04 \pm 6.14$ \\
LAD2 (mm)*** & $47.82 \pm 5.28$ & $44.93 \pm 5.19$ \\
RAD (mm)* & $45.85 \pm 4.46$ & $44.92 \pm 5.13$ \\
\hline
\end{tabular}

AOD: aortic diameter,

LAD1: left atrial parasternal diameter,

LAD2: left atrial apex diameter,

RAD: right atrial apex diameter.

$*: \mathrm{p}<0.05, * *: \mathrm{p}<0.01, * * *: \mathrm{p}<0.001$.

LVLDD decreased very slightly (4.1\%). Similarly slight but significant reductions were seen in the left atrial diameters measured in the parasternal and in the longitudinal views and in the RAD, representing 4.6, 6.0 and $2.0 \%$, respectively.

Table $\mathrm{V}$ displays the changes in the diastolic function parameters. We observed a marked, significant increase $(11.7 \%)$ in the early diastolic peak velocity (E), a slight, non-significant decrease in the late diastolic peak velocity (A). As the result of these changes, E/A ratio displayed a very marked, significant increase (16.4\%).

In the IVRT a slight but not significant decrease was seen.

The echocardiographic examinations did not reveal valve-deformities or abnormal flow in any cases, either at the first, or the second examinations.

Table V

Doppler-echocardiographic data before and after weight reduction (mean \pm S.D.)

\begin{tabular}{lcc}
\hline & & $(n=21)$ \\
& Before & After \\
\hline Doppler E* & $0.77 \pm 0.15$ & $0.86 \pm 0.15$ \\
Doppler A & $0.68 \pm 0.14$ & $0.65 \pm 0.13$ \\
E/A** & $1.16 \pm 0.23$ & $1.35 \pm 0.29$ \\
IVRT & $98.58 \pm 22.9$ & $93.97 \pm 17.86$ \\
\hline
\end{tabular}

E: early diastolic peak filling velocity,

A: late diastolic peak filling velocity,

E/A: ratio of early and late peak velocity,

IVRT: isovolumetric relaxation time

$*: \mathrm{p}<0.05, * *: \mathrm{p}<0.01, * * *: \mathrm{p}<0.001$. 


\section{Discussion}

In the present work an attempt was made to investigate the effect of a half-year life-style-modification program on the morphological and functional characteristics of the heart in obese women.

In comparison with data of other longitudinal echocardiographic studies $(6,8,12$, 15), we observed slightly increased left atrial and left ventricular measures and a reduced $\mathrm{E} / \mathrm{A}$ quotient in our obese patients in the beginning of our investigations.

According to several other data, increased cardiac dimensions, primarily a significant increase in left ventricular mass, may enhance the irritability of the left ventricle $(24,26,27,49,51)$. It is a fact that a pathologically increased left ventricular mass is a strong risk factor of sudden cardiac death syndrome even by itself $(1,19,20$, 23). Reduced early diastolic velocity (E) and increased late diastolic velocity (A), consequently a reduced E/A quotient, are signs for an impaired diastolic function of the heart $(10,18,28)$.

Data of the present study indicate that the half-year life-style-modification program brought about not only a marked weight loss, but also a significant improvement in both the morphological and functional characteristics of the heart. In this respect, our results are very similar to those of several other authors $(5,8,15,26$, $27,29,30,48,50)$.

Among the left ventricular morphologic characteristics, the decrease in the wall thickness was much more marked than at in the internal diameters, so the proportion of the left ventricular wall thickness to the internal diameter corresponded to the proportion (36) found in adult healthy women (36.5 vs. $34.6 \%$ ).

The calculated LVM displayed a very marked decrease, the $18.8 \%$ decrease highly exceeded that of body mass (5.9\%). According to the principle that cardiac dimensions should only be related to body dimensions having the same exponent (13, $14,33,34)$, comparisons can be made for the $\mathrm{LVM} / \mathrm{BM}$ or for the $\mathrm{LVM} / \mathrm{BSA}^{3 / 2}$ ratio. The $2.34 \mathrm{LVM} / \mathrm{BM}$ ratio decreased to 2.02 , the $\mathrm{LVM} / \mathrm{BSA}^{3 / 2}$ quotient decreased from 76.27 to 64.62 ; the lower values are within the physiological range (32).

Another favourable aspect is that the morphologic changes were concomitant to a meaningful functional improvement. In the systolic function, as characterised by EF, a very small, nonsignificant increase was seen. This can be explained by the fact that EF was within the normal range also before the training period. Diastolic function, however, displayed a very marked and significant improvement: the initially nearly pathologically decreased E/A quotient returned to the physiological range (12) so weight loss and the decrease of cardiac mass might induce a substantial improvement in the left ventricular compliance. 
Because of the significant increase in the left ventricular mass, and the diastolic dysfunction characteristic of obese patients, it is of importance that extremely obese patients should undergo early and thorough cardiologic studies (ECG-Holter, ABPM, echocardiography, and physical-stress-ECG). It is often difficult to carry out echocardiography in extreme obesity $(8-10 \%)$; nevertheless, information obtained in this way is of exceptional value.

Relying on the literature and on our own observations we are convinced that obese patients should enter a weight-reducing program at the earliest possible time in order to prevent or reduce the deleterious changes. Therapeutic weight reduction with dietary control and programmed exercise has evidenced its efficiency. The benefits quite certainly achieved are a reduction of left ventricular mass, of left ventricular wall thickness as well as of the size of the left and right atrium. It is also well known that regular physical exercise improves diastolic function $(3,5,6,10,18,29,31)$.

In addition to weight reduction, the treatment of extremely obese patients may extend to administering drugs that have been confirmed to reduce the extent of left ventricular hypertrophy, thus improving overall left ventricular function (ACEinhibitors, calcium-channel antagonists, beta-receptor antagonists and diuretics) (41, 42, $43,46)$. These, as well as programmed exercise, help relieve obese patients also from sympathetic nervous dominance.

On the basis of our results we can conclude that our patients achieved a $5.85 \%$ weight loss and managed to maintain it for half a year.

\section{Conclusions}

1. Eccentric left ventricular hypertrophy and diastolic dysfunction were observed at a relatively early stage in obese patients. Depending on the extent and duration of obesity the systolic function of the left ventricle deteriorates at a later stage.

2. The above-mentioned pathological changes appeared to be reversible for a relatively long time, especially when other diseases were not accompanied by obesity.

3. Echocardiographic parameters undergo favourable changes as a result of the complex conditioning program.

4. Improvement in the echocardiographic parameters may at the earliest be expected after half a year of controlled therapy. 


\section{Abbreviations}

AOD:

aortic diameter

LAD1:

left atrial parasternal diameter

LAD2:

left atrial apex diameter

IVSDT:

interventricular septal diastolic thickness

LVPWDT: left ventricular posterior wall diastolic thickness

LVIDD:

left ventricular internal diastolic diameter

LVLDD: left ventricular longitudinal diastolic diameter

RAD:

$\mathrm{EF}$ :

LVM: right atrial apex diameter

E:

ejection fraction

A:

ventricular mass

E/A early diastolic peak filling velocity late diastolic peak filling velocity

IVRT: ratio of early and late peak velocity isovolumetric relaxation time

\section{REFERENCES}

1. Abbott, R. D., Behrens, G. R., Sharps, D. S., Rodriguez, B. L.: Body mass index and thromboembolic stroke in nonsmoking men in older middle age. The Honolulu Heart Program. Stroke 25, 2370-2376 (1994).

2. Alpert, M. A., Hashimi, M. W.: Obesity and the heart. Am. J. Med. Sci. 306, 117-123 (1993).

3. Appleton, C. P., Hatle, L. K., Popp, R. L.: Relation of transmitral flow velocity patterns to left ventricular diastolic function: New insights from a combined hemodynamic and Doppler echocardiographic study. J. Am. Coll. Cardiol. 12, 426-440 (1988).

4. Ashwell, M.: Obesity in men and women. Int-J-Obes-Relat-Metab-Disord. 18, Suppl. 1 S1-7 (1994).

5. Baglivo, H., Fabrigues G.: Effect of moderate physical training on left ventricular mass in mild hypertensive persons. Hypertension 15, Suppl. 1, 153-156 (1990).

6. Bryg, R. J., Williams, G. A., Labowitz, A. J.: Effect of ageing on left ventricular diastolic filling in normal subjects. Am. J. Cardiol. 59, 971-974 (1987).

7. Buermann, B., Tremblay, A.: Effects of exercise training on abdominal obesity and related metabolic complications. Sports Med. 21, 191-212 (1996).

8. Chang-Sheng Ku, Shoa-Lin, Dang-Jiang Wang, Shi-Kun Chang, Wen-June Lee: Left ventricular filling in young normotensive obese adults. Am. J. Cardiol. 73, 613-615 (1994).

9. Devereux, R. B., Reichek, N.: Echocardiographic determination of left ventricular mass in man: anatomic validation of method. Circulation 55, 613-618 (1977).

10. Douglas, P. S., O'Toole, M.: Ageing and physical activity determine cardiac structure and function in the older athlete. J. Appl. Physiol. 72, 1969-1973 (1992).

11. Drewonski, A.: Progress in Obesity Research. Libbey. USA 459-464 (1990).

12. Feigenbaum, H.: Echocardiography - 5th ed. Lea and Febiger Philadelphia (1994).

13. George, K. P., Gates, P. E., Birch, K. M., Campbell, I. G.: Left ventricular morphology and function in endurance-trained female athletes. J. Sports Sciences 17, 633-642 (1999). 
14. George, K. P., Gates, P. E., Whyte, G., Fenoglio, R. A., Lea, R.: Echocardiographic examination of cardiac structure and function in elite cross trained male and female Alpine skiers. Br. J. Sports Med. 33, 93-98 (1999).

15. Grossmann, E., Oren, S., Messerli, F. H.: Left ventricular filling in the systemic hypertension of obesity. Am. J. Cardiol. 68, 57-60 (1991).

16. Halford, J. C. G., Heal, D. J., Blundell, J. E.: Effects in the rat of sibutramin on food intake and the behavioural satiety sequence. Br. J. Pharmacol. 114, 387 (1995).

17. Hammond, I. W., Devereux, R. B.: Relation of blood pressure and body build to left ventricular mass in normotensive and hypertensive employed adults. J. Am. Coll. Cardiol. 12, 996-1004 (1988).

18. Hatle, C.: Doppler ultrasound in cardiology. Lea and Febiger, Philadelphia (1988).

19. Hiroyasu, I., Masahiko, K., Mitsunori, D.: Left ventricular mass and subsequent blood pressure changes among middle-aged men in rural and urban Japanese populations. Circulation 89, 1717-1724 (1994).

20. Hughes, D., McGuire, A.: A review of the economic analysis of obesity. Br. Med. Bull. 53, 253-263 (1997).

21. James, W. P. T., Astrup, A., Finer, N., Kopelman, P. et al.: Sibutramine trial of obesity reduction and maintenance (STORM). Int. J. Obes. 22, Suppl. 3667 (1998).

22. Jákó, P., Alberti, E., Hertzka, P., Sidó, Z., Szamosi, K., Veress, J., Vigyázó, Gy.: Effect of diet and physical activity on the treatment of obese (in Hungarian, abstract in English) Hung. Rev. Sports Med. 31, 49-56 (1990).

23. Joanne, E., Manson, M. D., Walter, C., Willet, M. D., Meir, J., Stampfer, M. D., Graham, A., Colditz, M. B., David, J., Hunter, M. B., Susan, E. Hankinson, C. H. Hennekens, F. E. Speizer: Body weight and mortality among women. The N. Engl. J. of Med. 333, 677-685 (1995).

24. Katz, A. M. Cardiomyopathy of overload: a major determinant of prognosis in congestive heart failure. The N. Engl. J of Med. 322, 100-110 (1990).

25. Kopelmann, P. G., Stock, M. J.: Clinical obesity. Blackwell Science Ltd. (1998).

26. Krumholz, H. M., Larson, M., Levy, D.: Prognosis of left ventricular geometric patterns in the Framingham Heart Study. J. Am. Coll. Cardiol. 25, 879-884 (1995).

27. Lauer, M. S., Anderson, K. M., Kannel, W. B., Levy, D.: The impact of obesity on left ventricular mass and geometry. The Framingham Heart Study. JAMA 266, 231-236 (1991).

28. Levy, D., Garrison, R. J.: Prognostic implications of echocardiographically determined left ventricular mass in the Framingham Heart Study. The N. Engl. J. of Med. 322, 1561-1566 (1990).

29. Levy, W. C., Cerquiera, M. D., Abrass, I. B., Schwartz, R. S., Stratton, J. R.: Endurance exercise training augments diastolic filling at rest and during exercise in healthy young and older men. Circulation 88, 116-126 (1993).

30. MacMahon, W. S., Wicken, E. L. D., Macdonald, J. G.: The effect of weight reduction on left ventricular mass. The N. Engl. J. of Med. 314, 334-339 (1986).

31. Matsuda, M., Sugishita, Y., Koseki, S., Ito, I., Akatsuka, T., Takamtso, K.: Effect of exercise on left ventricular diastolic filling in athletes and non-athletes. J. Appl. Physiol. 55, 323-328 (1983).

32. Pavlik, G., Olexó, Zs., Bánhegyi, A., Sidó, Z., Frenkl, R.: Gender differences in the echocardiographic characteristics of the athletic heart. Acta Physiol. Hung. 86, 273-278 (1999).

33. Pavlik, G., Olexó, Zs., Frenkl, R.: Echocardiographic estimates related to various body size measures in athletes. Acta Physiol. Hung. 84, 171-181 (1996).

34. Pavlik, G., Olexó, Zs., Petrekanits, M., Osváth, P.: Arithmetic calculation with echocardiographic indices (in Hungarian, abstract in English). Hung. Rev. Sports Med. 36, 115-129 (1995).

35. Pavlik, G., Olexó, Zs., Sidó, Z., Frenkl, R.: Doppler-echocardiographic examinations in the assessment of the athletic heart. Acta Physiol. Hung. 86, 7-22 (1999).

36. Pelliccia, A., Maron, B. J., Culasso, F., Spataro, A., Caselli, G.: Athlete's heart in women. Echocardiographic characterization of highly trained female athletes. JAMA 276, 211-215 (1996).

37. Saris, W. H.: Physical inactivity and metabolic factors as predictors of weight gain. Nutr. Rev. 54, $110-115$ (1996). 
38. Sasson, Z., Rasooly, Y.: Insulin resistance is an important determinant of left ventricular mass in the obese. Circulation 88, 1431-1436 (1993).

39. Schiller, N. B., Skioldebrand, C. G., Schiller, E. J.: Canine left ventricular mass estimation by twodimensional echocardiography. Circulation 68, 210-216 (1983).

40. Shapiro, L. M.: Physiological left ventricular hypertrophy. Br. Heart J. 52, 135-138 (1984).

41. Sidó, Z.: Conditioning program in the treatment of obesity. Hung. Rev. Sports Med. 39, 133-138 (1998).

42. Sidó, Z., Jákó, P., Martos, É., Ékes, E., Haász, P.: Echocardiography in obese patients. Hung. Med. J. 136, 2667-2671 (1995).

43. Sidó, Z., Jákó, P., Martos, É., Haász, P.: Impedance cardiography in obese patients. Hung. Rev. Sports Med. 35, 143-149 (1994).

44. Sidó, Z., Jákó, P., Martos, É., Haász, P.: Obesity and stroke. Hung. Rev. Sports Med. 37, 203-213 (1996).

45. Sidó, Z., Martos, É., Jákó, P., Ékes, E., Haász, P.: Comparative echocardiography of normotensive and hypertensive obese patients. Hung. Rev. Sports Med. 2, 105-113 (1995).

46. Sidó, Z., Jákó, P., Pucsok, J., Martos, É.: Effects of the conditioning onto obese diabetic patients. Hung. Rev. Sports Med. 37, 1 31-40 (1996).

47. Sidó, Z., Veress, J., Jákó, P.: Exercise testing of obese patients. Hung. Rev. Sports Med. 31, 41-47 (1990).

48. Stephen, W., MacMahon, D., Wilcken, E. L., Macdonald, G. J.: The effect of weight reduction on left ventricular mass. A randomized controlled trial in young, overweight hypertensive patients. The N. Eng. J. of Med. 314, (1986).

49. Sullivan, J. M., Vander Zwaag, R., el-Zeky, F. et al.: Left ventricular hypertrophy: effect on survival. J. Am. Coll. Cardiol. 22, 508-513 (1993).

50. Van Gaal, L., Wauters, M., De Leeuw, I.: The beneficial effects of modest weight loss on cardiovascular risk factors. Int. J. of Obesity 21, suppl. 1 S5-S9 (1994).

51. Vogt, M., Motz, W. H.: Pathophysiology and clinical aspects of hypertensive hypertrophy. Eur. Heart J. 14, Supplement D 2-7 (1993) 